\title{
Electrical distribution system reconfiguration for power loss reduction by the Salp Swarm algorithm
}

\author{
Daranpob Yodphet ${ }^{\mathrm{a}}$, Arun Onlam ${ }^{\mathrm{a}}$, Apirat Siritaratiwat ${ }^{\mathrm{a}}$, and Pirat Khunkitti ${ }^{\mathrm{a} *}$ \\ ${ }^{a}$ Department of Electrical Engineering, Faculty of Engineering, Khon Kaen University, Khon Kaen 40002, Thailand
}

\begin{abstract}
A novel optimization algorithm called the Salp Swarm Algorithm (SSA) has recently been found to be effective for solving optimization problems; however solving optimal system reconfiguration using SSA has not been reported in the literature. Thus, this paper proposes a network reconfiguration of an electrical distribution system based on the SSA for power loss reduction. The 33 and 69 bus distribution systems are focused on for the network reconfiguration. Simulation results obtained by SSA are compared with those of the Genetic Algorithm and Simulated Annealing, which are well-known algorithms. It is found that the performance of an electrical distribution system reconfigured by SSA is reconfigured better than by other methods, in terms of the quality of the solutions and the average elapsed time. Thus SSA becomes a good choice for power loss reduction in an electrical distribution system.
\end{abstract}

Keywords: Network reconfiguration, Power loss reduction, Heuristic algorithm, Salp swarm algorithm

\section{Introduction}

As the electricity demand of the power distribution system has been continually increasing with the incessant growth of population, the characteristics of the load will be frequently altered according to customer behavior [1]. Constructions of supplementary power distribution systems are required. However the large-scale of a power system typically yields a massive power loss, which is one of the most important problems for an electrical system. Many approaches have been proposed in the literature in order to minimize the power loss of an electrical system. One of the most effective techniques is network reconfiguration, because of its simple implementation and low-cost investment. Generally, the electrical distribution system consists of two types of switches, which are tie switches and sectionalizing switches. The status of tie switches is normally open while that of sectionalizing switches is normally closed. The topology of a system can be determined by the statuses of tie and sectionalizing switches. System reconfiguration is the process of changing the topology of the distribution network by altering the statuses of switches.

Several methods have been proposed for solving the optimal reconfiguration problems for power loss reduction. A heuristic method has been a general technique for solving optimal reconfiguration problems [2-4]; it is an optimization process which can find an estimated optimal solution [5]. The outstanding property of this approach is simple implementation. Recently the meta-heuristic method has been widely developed from the heuristic method to optimize electrical system reconfiguration. It performs a recursive process that allows the searching process to efficiently find the near-optimal solution using learning strategies and intelligently combining different concepts [5]. The advantage of this approach is its ability to solve a wide range of optimization problems by focusing only on inputs and outputs [6]. Several metaheuristic methods have been proposed in the literature to achieve the minimum power loss of power systems, i.e. Genetic Algorithm (GA), Evolution Programming (EP), Ant Colony Search Algorithm

\footnotetext{
* Manuscript received September 10, 2018; revised January 12, 2018.

Corresponding author. Tel.: +66-86-636-5678; E-mail address: piratkh@kku.ac.th.

doi: $10.12720 /$ sgce.8.2.156-163
} 
(ACSA), Tabu Search (TS), Simulated Annealing (SA), Harmony Search Algorithm, Artificial Bee Colony (ABC) and Particle Swarm Optimization (PSO) [7-16]. More recently, the methodologies based on the Bacterial Foraging Optimization Algorithm (BFOA), Fireworks Algorithm (FA), Cuckoo Search Algorithm (CSA) and Runner Root Algorithm (RRA) have become effective methods for solving system reconfiguration problems [17-20]. However, the performance of these existing methods, such as the convergence property or the computation time can still be improved.

Recently, a new meta-heuristic method called the Salp Swarm Algorithm (SSA) has been proposed to solve optimization problems. It was inspired by the swarming behavior of salps when navigating and foraging in the ocean. In particular, the SSA indicates outstanding performance at finding the optimal solution of optimization problems. However, the reconfiguration of electrical distribution systems using SSA has not yet been reported.

This paper aims to solve the optimal system reconfiguration for power loss reduction using the SSA in the 33 and 69 bus distribution systems. The parameters indicating the algorithm performance, including the best power loss, average power loss, average elapsed time and standard deviation are analyzed. Simulation results obtained by SSA are compared with those of the SA and GA methods, which are wellknown algorithms.

\section{Problem Formulation}

The purpose of an electrical distribution system reconfiguration problem is to find an optimal topology of a radial network for power loss reduction without violating the constraints. The reconfiguration problem constraints are indicated as follows: all loads must be served, the radial structure of distribution system must be maintained, bus voltage should be between its upper and lower limits and the branch current should be maintained within its range. The objective function for the minimization of power loss can be expressed as follows [14]:

$$
\text { Minimize } O b j=\sum_{i=1}^{n b} r_{i} \frac{\left(P_{i}^{2}+Q_{i}^{2}\right)}{V_{i}^{2}}
$$

which is subject to the following constraints:

- No load can be left out of service,

- Radial system structure must be retained, $\operatorname{det}(A)=1$ or $-1 \quad($ radial system $)$

- Bus voltage should be between upper and lower limits, $V^{\min } \leq\left|V_{j}\right| \leq V^{\max }$

- Branch current should be within the proposed range,

$0 \leq\left|I_{i}\right| \leq I_{i}^{\max }$

Here $O b j$ is the objective function $(\mathrm{kW}), n b$ is the total number of branches, $r_{\mathrm{i}}$ is the resistance of a branch $i, P_{\mathrm{i}}$ is the active power at the sending end of branch $i, Q_{\mathrm{i}}$ is the reactive power at the sending end of branch $i, V_{\mathrm{i}}$ is the voltage at the sending end of branch $i, A$ is the bus incidence matrix, $V^{\min }$ is the bus minimum voltage limit ( 0.9 p.u.), $\left|V_{\mathrm{j}}\right|$ is the voltage magnitude of the bus $j, V^{\max }$ is the bus maximum voltage limit (1.0 p.u.), $\left|I_{\mathrm{i}}\right|$ is the current magnitude of branch $i$ and $I_{\mathrm{i}}^{\text {max }}$ is the maximum current limit of branch $i$.

\section{Overview of Salp Swarm Algorithm}

The SSA is a new nature-inspired technique for solving optimization problems. The merit of this algorithm is its ability to solve real-world problems with difficult and unknown search spaces [6]. The SSA imitates the behaviors of salps when they form a swarm called a salps chain [6]. Salps in the chain 
are divided to two groups which are leaders and followers. The salp at the front of the chain is the leader and the rest of the salps are followers. The leader leads the swarm and the followers follow each other. The position of salps is determined in a $k$-dimensional search space where $k$ is the number of variables of the given problem. So, the position of all the salps are stored in a two-dimensional matrix called $x$ [6]. It is assigned that the position of the best salp is a food source, called $F$, which is chased by the salps chain. The food source will be updated during optimization, because the salps chain will find a better solution by exploring and exploiting the space around it [6]. Thus, the salps chain has to move towards the global optimal, which changes over the range of iterations. The mathematical model for moving the salps chain can be expressed as follows [6]:

- The position of the leader is

$$
x_{j}^{1}=\left\{\begin{array}{lll}
F_{j}+c_{1}\left(\left(u b_{j}-l b_{j}\right) c_{2}+l b_{j}\right) & \text { when } \quad c_{3} \geq 0.5 \\
F_{j}-c_{1}\left(\left(u b_{j}-l b_{j}\right) c_{2}+l b_{j}\right) & \text { when } \quad c_{3}<0.5
\end{array}\right.
$$

where $x_{\mathrm{j}}{ }^{1}$ is the position of the first salp (leader) in the $j$ th dimension, $F_{\mathrm{j}}$ is the position of the food source in the $j$ th dimension (the swarm's target), $u b_{\mathrm{j}}$ is the upper bound of the $j$ th dimension, $l b_{\mathrm{j}}$ is the lower bound of the $j$ th dimension; $c_{2}$ and $c_{3}$ are random numbers uniformly generated in the interval of $[0,1]$, and the coefficient $c_{1}$ is the main controlling parameter of the SSA, defined by

$$
c_{1}=2 e^{-\left(\frac{4 l}{L}\right)^{2}}
$$

where $l$ is the current iteration and $L$ is the maximum number of iterations.

- The position of the follower is

$$
x_{j}^{i}=\frac{1}{2}\left(x_{j}^{i}+x_{j}^{i-1}\right)
$$

where $i \geq 2$ and $x_{\mathrm{j}}^{\mathrm{i}}$ is the position of the $i$ th follower salp in the $j$ th dimension.

\section{Implementation of the Salp Swarm Algorithm for Distribution System Reconfiguration}

In this work, the SA, GA and SSA methods are performed on the 33 bus [8] and 69 bus [21] distribution systems. The 33 bus system is shown in Fig. 1. These algorithms are programmed using MATLAB and run on a computer with Intel Core i7-7700-3.60 GHz and 16 GB DDR4 RAM.

The procedures for the distribution system reconfiguration performed by SSA are defined as follows.

- Step 1: Read the parameters of the distribution system and initialize the maximum number of iterations.

- Step 2: Initialize the salp population by considering upper and lower bounds, with each salp indicated as the position of tie switches.

- Step 3: Calculate the fitness of initial salps by using the following equation:

Minimize Fitness $=\left\{\begin{array}{c}\text { Obj when the constraints are not violated. } \\ \text { Obj }+ \text { Penalty when the constraints are violated. }\end{array}\right.$

where $O b j$ is the objective function $(\mathrm{kW})$ from equation (1), and Penalty is a large constant.

- Step 4: Initialize $F$ (the swarm's target) which is the position of the best salp (best fitness).

- Step 5: Initialize iteration of 2, which is started from the second iteration since the first iteration was dedicated to calculate the fitness of salps.

- Step 6: Update $c_{1}$ using equation (6).

- Step 7: Update the position of the leading salp using equation (5).

- Step 8: Update the position of the follower salps using equation (7).

- Step 9: Amend the salps based on the upper and lower bounds. 


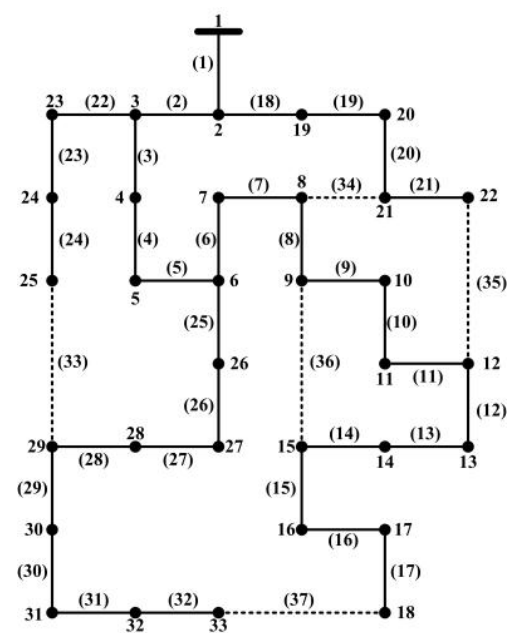

Fig. 1. The 33-bus distribution system [8].

- Step 10: Calculate the fitness of each salp using equation (8).

- Step 11: Update $F$.

- Step 12: Update the current iteration (iteration = iteration +1 ).

- Step 13: Check the program interrupting condition, if iteration < the maximum number of iterations, go to step 6, otherwise, go to step 14.

- Step 14: End.

The SSA has been implemented and applied to the network reconfiguration in 33 and 69 bus distribution systems. The focused parameters include the best power loss, average power loss, average elapsed time and standard deviation. Simulation results obtained by SSA are compared with those of the SA and GA methods.

\section{Test Results}

\subsection{Optimization of parameter setting of SSA}

First of all, in order to maximize the performance of SSA, the reconfiguration problem was repeatedly solved by 100 runs with different values of main controlling parameter, $c_{1}=2 e^{-\left(\frac{X l}{L}\right)^{2}}$, from 0.5 to 8 [6]. The average power loss and elapsed time of 33 and 69 bus reconfigured system are indicated in Figs. 2(a) and 2(b), respectively. It is found that using $X=2$ indicates the optimal value between average power loss and average elapsed time, then we set $X=2$ in the simulations using SSA.

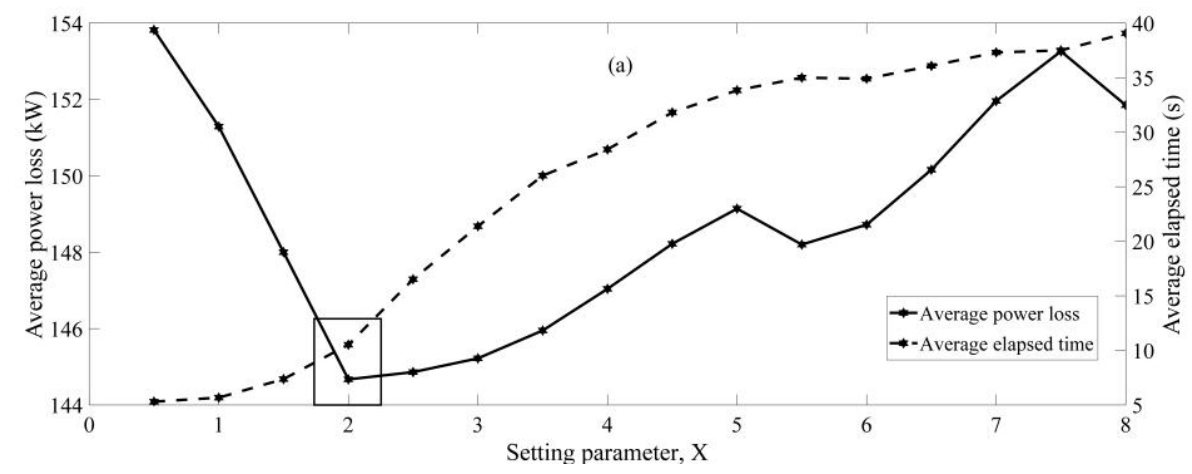




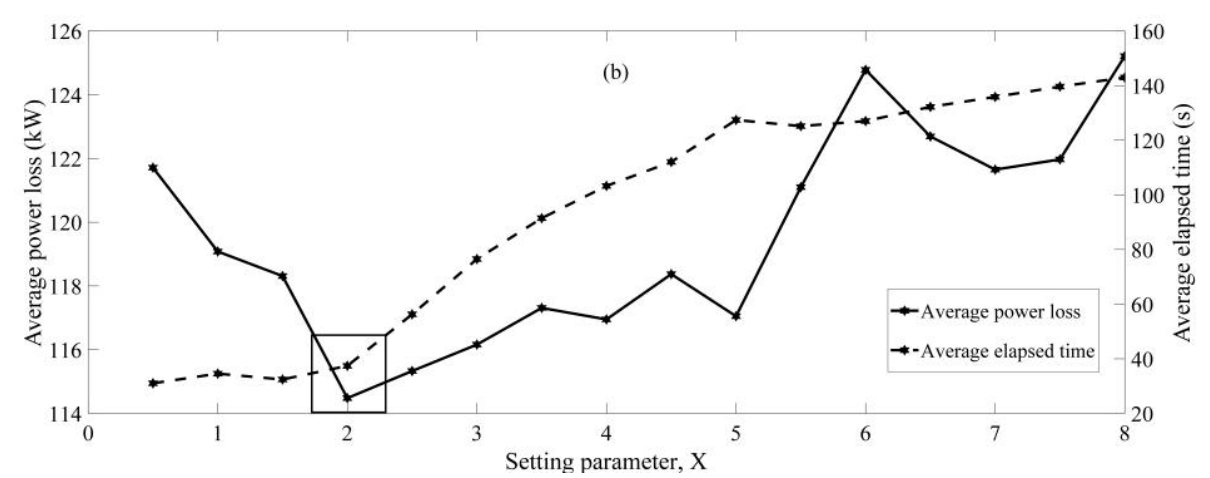

Fig. 2. The average power loss and elapsed time of the reconfigured system with varying setting parameters, $X$, of SSA in (a) 33 bus system and (b) 69 bus system.

\subsection{Network reconfiguration of 33 bus distribution system}

As shown in Fig. 1, the first example is the 33 bus radial system, which comprises 37 branches and 5 tie switches. It was described in the paper of J. Z. Zhu, where the system data are also given [8]. The five initial tie switches of 33, 34, 35, 36 and 37 were set. Meanwhile, the initial power loss was set as 202.68 $\mathrm{kW}$. The parameters of SA, GA and SSA are defined as follows:

- The SA parameters: iteration is 200 , sub-iteration is 15 , initial temperature is 1000 and temperature reduction ratio is 0.95 .

- The GA parameters: population is 20 , iteration is 200 , crossover rate is 0.50 and mutation rate is 0.03 .

- The SSA parameters: population is 20 , iteration is 200 and $c_{1}$ is adopted from equation (6) as $2 e^{-\left(\frac{2 l}{L}\right)^{2}}$

In the reconfiguration of the 33 bus distribution system, the SA, GA and SSA were repeatedly solved over 100 runs. The results of best, average and worst loss among the 100 runs are shown in Table 1 . The average elapsed time and standard deviation are also indicated.

Table 1. Simulation results of 33 bus distribution system

\begin{tabular}{|c|c|c|c|c|}
\hline \multirow[t]{2}{*}{ Item } & \multirow{2}{*}{$\begin{array}{c}\text { Initial } \\
\text { configuration }\end{array}$} & \multicolumn{3}{|c|}{ After reconfiguration } \\
\hline & & SA & GA & SSA \\
\hline Tie switches & $33,34,35,36,37$ & $\begin{array}{l}7,9,14,32,33 \\
\text { (best solution) }\end{array}$ & $\begin{array}{l}7,9, \quad 14, \quad 32, \quad 33 \\
\text { (best solution) }\end{array}$ & $\begin{array}{l}7,9,14,32,33 \\
\text { (best solution) }\end{array}$ \\
\hline Best power loss $(\mathrm{kW})$ & 202.68 & 139.55 & 139.55 & 139.55 \\
\hline Worst power loss $(\mathrm{kW})$ & - & 189.39 & 159.69 & 157.44 \\
\hline Average power loss (kW) & - & 150.61 & 149.05 & 144.67 \\
\hline Average loss reduction (\%) & - & 25.69 & 26.46 & 28.62 \\
\hline Average elapsed time (s) & - & 22.81 & 19.54 & 10.52 \\
\hline Standard deviation & - & 12.95 & 4.28 & 3.38 \\
\hline
\end{tabular}

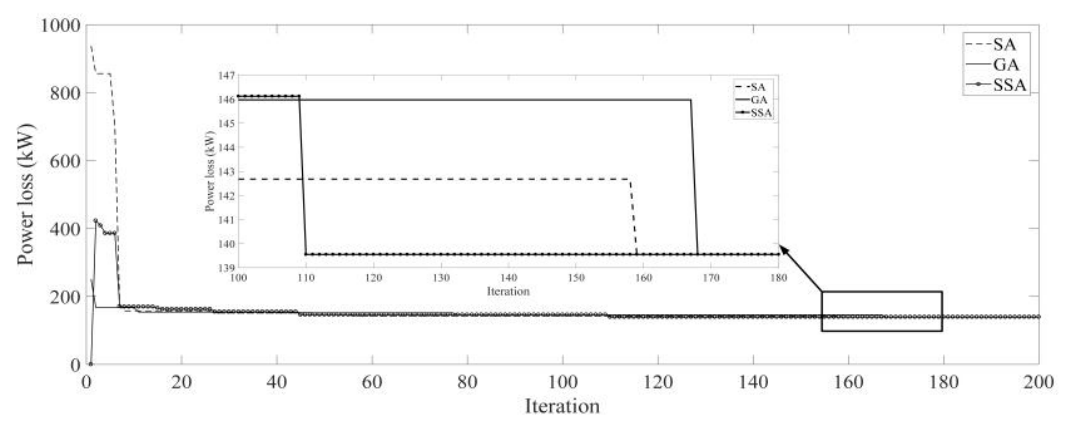

Fig. 3. Convergence characteristics of SA, GA and SSA for 33 bus system. 
From the simulation results shown in Table 1, it is observed that the best power loss for the distribution system has the same values of SA, GA and SSA. However, the worst power loss obtained by SSA is less than that obtained by the other algorithms. As a result, the system reconfigured by SSA indicates better average power loss than the others, which yields the best percentage of average loss reduction. In addition, the average elapsed time and standard deviation accomplished by SSA are significantly less than those of SA and GA. Thus it is concluded that the overall performance of the electrical distribution system reconfigured by SSA is remarkably better than those of SA and GA. In addition, the convergence characteristic of the SSA compared with that of SA and GA for the 33 bus distribution system is illustrated in Fig. 3. It is found that the SA, GA and SSA could achieve the best solution within 159, 168 and 110 iterations, respectively. Thus, the SSA also indicates faster convergence performance compared to other methods.

\subsection{Network reconfiguration of 69 bus distribution system}

The second example is the 69 bus distribution system which comprises 73 branches and 5 tie switches [21]. The five initialized tie switches are 69, 70, 71, 72, and 73. Meanwhile, the initial power loss is $225.00 \mathrm{~kW}$. Parameters of SA, GA and SSA are defined as follows:

- The SA parameters: iteration is 300 , sub-iteration is 15 , initial temperature is 5000 and temperature reduction ratio is 0.99 .

- The GA parameters: population is 30 , iteration is 300 , crossover rate is 0.50 and mutation rate is 0.03 .

- The SSA parameters: population is 30 , iteration is 300 and $c_{1}$ is adopted from equation (6) as $2 e^{-\left(\frac{2 l}{L}\right)^{2}}$.

In this example, Table 2 shows the best, average and worst results among the 100 runs. The average elapsed time and standard deviation are also illustrated.

Table 2. Simulation results of 69 bus distribution system

\begin{tabular}{|c|c|c|c|c|}
\hline \multirow[t]{2}{*}{ Item } & \multirow{2}{*}{$\begin{array}{c}\text { Initial } \\
\text { configuration }\end{array}$} & \multicolumn{3}{|c|}{ After reconfiguration } \\
\hline & & SA & GA & SSA \\
\hline Tie switches & $69,70,71,72,73$ & $\begin{array}{l}14,58,61,69,70 \\
\text { (best solution) }\end{array}$ & $\begin{array}{l}14,58,61,69,70 \\
\text { (best solution) }\end{array}$ & $\begin{array}{l}14,58,61,69,70 \\
\text { (best solution) }\end{array}$ \\
\hline Best power loss $(\mathrm{kW})$ & 225.00 & 99.62 & 99.62 & 99.62 \\
\hline Worst power loss $(\mathrm{kW})$ & - & 327.24 & 132.58 & 136.17 \\
\hline Average power loss (kW) & - & 167.29 & 114.68 & 114.48 \\
\hline Average loss reduction (\%) & - & 25.64 & 49.03 & 49.12 \\
\hline Average elapsed time (s) & - & 52.41 & 64.69 & 37.41 \\
\hline Standard deviation & - & 65.94 & 6.65 & 8.31 \\
\hline
\end{tabular}

From Table 2, it is seen that the SA, GA and SSA have the same best solution of power loss. However, the worst power loss obtained by SSA is significantly less than that obtained by SA. In particular, the SSA indicates the best average power loss reduction. Moreover, the average elapsed time obtained by SSA is remarkably less than those of the SA and GA. In addition, the SSA has better standard deviation than that of the SA. Thus, it can be concluded that the performance of the distribution system reconfigured by the SSA is notably better than those of SA and GA in terms of average power loss and average elapsed time.

From the above simulation results, it is seen that both of the distribution systems reconfigured by the SSA method have better performance than those of the SA and GA methods. The reason why the SSA is quite effective in solving optimization problems is because the SSA includes a procedure to update the position of the leading salp with respect to the food source (the swarm's target), which is the prefered solution so far, then the leader always explores and exploits the space around it [6], while the SA and GA update their solutions by a random distribution without a target. Therefore, the SSA has a better convergence property and less average elapsed time than SA and GA. 


\section{Conclusion}

In this research, the network reconfiguration of 33 and 69 bus electric distribution systems for power loss minimization using SSA are reported. The simulation results obtained by SSA are compared with those of SA and GA algorithms. It is found that the average power loss reduction of the electric distribution system reconfigured by SSA is higher than those of SA and GA for both 33 and 69 bus test systems. Also, the average elapsed time of SSA is remarkably less than those for SA and GA for both of the 33 and 69 bus test systems. Moreover, the standard deviation of SSA is significantly less than that of SA and GA for the 33 bus test system. From the results, it can be concluded that the performance of the SSA algorithm for the power loss minimization of an electrical distribution system is better than the SA and GA algorithms. Then, the SSA becomes the best choice to reduce the power loss in an electrical distribution system.

\section{Acknowledgements}

This work was financially supported by Provincial Electricity Authority (PEA).

\section{References}

[1] Zidan A, El-Saadany E. F. Distribution system reconfiguration for energy loss reduction considering the variability of load and local renewable generation. Energy, 2013; 59:698-707.

[2] Civanlar S, Grainger J.J, Yin H, Lee S. S. H. Distribution feeder reconfiguration for loss reduction. IEEE Transactions on Power Delivery, 1988; 3(3):1217-1223.

[3] Baran ME, Wu FF. Network reconfiguration in distribution systems for loss reduction and load balancing. IEEE Transactions on Power Delivery, 1989; 4(2):1401-1407.

[4] Shirmohammadi D, Hong HW. Reconfiguration of electric distribution networks for resistive line losses reduction. IEEE Transactions on Power Delivery, 1989; 4(2):1492-1498.

[5] Badran O, Mekhilef S, Mokhlis H, Dahalan W. Optimal reconfiguration of distribution system connected with distributed generations: a review of different methodologies. Renewable and Sustainable Energy Reviews, 2017; 73:854-867.

[6] Mirjalili S, Gandomi AH, Mirjalili SZ, Saremi S. Salp swarm algorithm: a bio-inspired optimizer for engineering design problems. Advances in Engineering Software, 2017; 114:163-191.

[7] Nara K, Shiose A, Kitagawa M, Ishihara T. Implementation of genetic algorithm for distribution systems loss minimum reconfiguration. IEEE Transactions on Power Systems, 1992; 7(3):1044-1051.

[8] Zhu J. Optimal reconfiguration of electrical distribution network using the refined genetic algorithm. Electric Power Systems Research, 2002; 62(1):37-42.

[9] Hsiao YT. Multiobjective evolution programming method for feeder reconfiguration. IEEE Transactions on Power Systems, 2004; 19(1):594-599.

[10] Su CT, Chang CF, Chiou JP. Distribution network reconfiguration for loss reduction by ant colony search algorithm. Electric Power Systems Research, 2005; 75(2):190-199.

[11] Chang CF. Reconfiguration and capacitor placement for loss reduction of distribution systems by ant colony search algorithm. IEEE Transactions on Power Systems, 2008; 23(4):1747-1755.

[12] Zhang D, Fu Z, Zhang L. An improved TS algorithm for loss-minimum reconfiguration in large-scale distribution systems. Electric Power Systems Research, 2007; 77(5-6):685-694.

[13] Jeon YJ, Kim JC, Kim JO, Lee KY, Shin JR. An efficient simulated annealing algorithm for network reconfiguration in largescale distribution systems. IEEE Transactions on Power Delivery, 2002; 17(4):1070-1078.

[14] Rao RS, Narasimham SVL, Raju MR, Rao AS. Optimal network reconfiguration of large-scale distribution system using harmony search algorithm. IEEE Transactions on Power Systems, 2011; 26(3):1080-1088.

[15] Rao RS, Narasimham SVL, Ramalingaraju M. Optimization of distribution network configuration for loss reduction using artificial bee colony algorithm. World Academy of Science, Engineering and Technology, International Journal of Electrical, Computer, Energetic, Electronic and Communication Engineering, 2008; 2(9):1964-1970.

[16] Olamaei J, Niknam T, Gharehpetian G. Application of particle swarm optimization for distribution feeder reconfiguration considering distributed generators. Applied Mathematics and Computation, 2008; 201(1-2):575-586. 
[17] Kumar KS, Jayabarathi T. Power system reconfiguration and loss minimization for distribution systems using bacterial foraging optimization algorithm.Electrical Power and Energy Systems, 2012; 36(1):13-17.

[18] Imran AM, Kowsalya M. A new power system reconfiguration scheme for power loss minimization and voltage profile enhancement using fireworks algorithm.Electrical Power and Energy Systems, 2014; 62:312-322.

[19] Nguyen TT, Truong AV. Distribution network reconfiguration for power loss minimization and voltage profile improvement using cuckoo search algorithm. Electrical Power and Energy Systems, 2015; 68:233-242.

[20] Nguyen TT, Nguyen TT, Truong AV, Nguyen QT, Phung TA. Multi-objective electric distribution network reconfiguration solution using runner-root algorithm. Applied Soft Computing, 2017; 52:93-108.

[21] Duan DL, Ling XD, Wu XY, Zhong B. Reconfiguration of distribution network for loss reduction and reliability improvement based on an enhanced genetic algorithm. Electrical Power and Energy Systems, 2015; 64:88-95. 